\title{
Eficiência e custo do controle químico da mancha de alternaria em tangor murcote
}

\author{
Adimara Bentivoglio Colturato ${ }^{1,2}$, Tatiana Paulossi, Wilson Story Venâncio ${ }^{3}$, Edson Luiz Furtado ${ }^{1,2}$
}

\begin{abstract}
${ }^{1}$ Departamento de Produção Vegetal/ Defesa Fitossanitária, Faculdade de Ciências Agronômicas (FCA)/UNESP, CP 237, CEP 18.603-970, Botucatu, São Paulo, Brasil; ${ }^{2}$ Bolsista do CNPq; ${ }^{3}$ Departamento de Fitotecnia e Fitossanidade, Universidade Estadual de Ponta Grossa (UEPG), Av. Carlos Cavalcanti, 4748, Uvaranas, cep 84030-900, Ponta Grossa - PR.

Autor para correspondência: Adimara B. Colturato (acolturato@fca.unesp.br)
\end{abstract}

Data de chegada: 10/07/2007. Aceito para publicação em: 13/03/2009

\section{RESUMO}

Colturato, A.B.; Paulossi, T.; Venâncio, W.S.; Furtado, E.L.. Eficiência e custo do controle químico da mancha de alternaria em tangor murcote. Summa Phytopathologica, v.35, n.3, p.210-215, 2009

A mancha de alternaria, causada por Alternaria alternata f. sp. citri, afeta tangelos Minneola, tangerinas Dancy, tangores Murcote e, menos freqüentemente, tangelos Orlando, tangerinas Novas, Lees e Sunburst. Esta doença causa desfolha grave, queda de frutos e manchas nas frutas. O objetivo deste trabalho foi estabelecer o melhor fungicida e a melhor dose para o controle da mancha marrom de alternaria. O delineamento experimental foi de parcelas subdivididas em blocos, com 10 tratamentos principais e 3 doses (subparcelas), com 5 repetições. Foram feitas 5 aplicações, com intervalo de 15 dias. Os tratamentos foram: azoxystrobin, pyraclostrobin, trifloxystrobin, trifloxystrobin + tebuconazole (2 aplicações) seguido de 3 aplicações de mancozeb, difenoconazole, trifloxystrobin + propiconazole, iprodione, trifloxystrobin + propineb (2 aplicações) seguido de 3 aplicações de oxicloreto de cobre, oxicloreto de cobre + óleo e testemunha. Simultaneamente foram feitas avaliações de incidência e número de lesões por folha. Ao surgimento dos frutos foram avaliadas a incidência em frutos e a produtividade em $\mathrm{Kg} / \mathrm{ha}$. Todos os tratamentos foram superiores à testemunha quanto a produtividade. Entre os produtos utilizados o tratamento com trifloxystrobin + propiconazole foi rentável comparandose custo e produtividade.

Palavras-chave adicionais: Alternaria alternata, programa de aplicação, fungicidas, custo e produtividade.

\section{ABSTRACT}

Colturato, A.B.; Paulossi, T.; Venâncio, W.S.; Furtado, E.L.. Efficiency and cost of chemical control of alternaria brown spot. Summa Phytopathologica, v.35, n.3, p.210-215, 2009

Alternaria brown spot, caused by Alternaria alternata sp. citri, attacks with more intensity the Tangelos Minneola, tangerine Dancy, and Murcotts, and with less intensity the tangelos Orlando and the tangerinas Novas, Lees and Sunburst. This disease causes severe defoliation and drop or necrotic spots in the fruits. The aim of this work was to evaluated the chemical control of brown spot, and to define the most appropriate dosage of fungicide to control it. The experimental design was split-spot, with ten treatments and 3 doses of fungicides, with five replicates, the fungicides were: azoxystrobin, pyraclostrobin, trifloxystrobin, trifloxystrobin + propinebe ( 2 applications) followed by 3 applications of mancozeb, difenoconazole, trifloxystrobin + propiconazole, iprodione, trifloxystrobin + tebuconazole $(2$ applications) followed by 3 applications of copper, copper + oil and control without fungicide. It was evaluated the incidence and number of lesions per leaf, as well the incidence of the disease on fruits and yield at the time of fruits appearing $(\mathrm{Kg} / \mathrm{ha})$. All treatments were superior to the control for yield. Among them, the treatment with trifloxystrobin + propiconazole was more profitable comparing cost and yield.

Keywords: Alternaria alternata, spray program, fungicides, cost, productivity.

A mancha marrom de alternaria (MMA), causada por Alternaria alternata f. sp. citri, afeta o tangor Murcote causando lesões em folhas, ramos e frutos, acarretando em desfolha e manchas que depreciam o fruto comercialmente. Esta doença foi constatada no Brasil a partir de 2001. O ataque severo pode acarretar em queda de folhas e de frutos e em manchas nos frutos reduzindo a produção (2).

O fungo produz uma toxina específica para o hospedeiro que é responsável pelas lesões necróticas. A toxina é, as vezes, translocada no sistema vascular, produzindo clorose e necrose que se estendem ao longo das lesões, freqüentemente resultando em queda de folhas, de frutos e seca de ponteiros. Áreas necrosadas podem ser rapidamente colonizadas por Colletotrichum gloesporioides, e corpos de frutificação aparecem nos círculos concêntricos. O tamanho da lesão e a extensão da necrose está diretamente relacionado com a suscetibilidade do hospedeiro (8).

Modificações nas práticas culturais ajudam a reduzir o impacto desta doença, mas o meio de controle mais utilizado é por aplicações de fungicidas; porém a mancha marrom é uma doença de difícil controle podendo ser necessárias de 7 a 10 aplicações (9).

Nos anos de 1993 e 1994, na África do Sul, programas para controle da mancha marrom de alternaria em tangelo Minneola foram feitos comparando oxicloreto de cobre, mancozeb, maneb mais óxido de zinco, procimidone, iprodione e tebuconazole. 
Mancozeb, aplicado com intervalos de 2 semanas, foi o mais efetivo no programa de pulverização, seguido em ordem de eficiência por procimidone, maneb mais óxido de zinco e mancozeb aplicado em intervalos de 4 e 3 semanas. Iprodione e tebuconazole não foram particularmente efetivos, embora tenham se mostrado significantemente melhores do que a testemunha (6).

Solel et al. (4) avaliando proteção foliar em Israel, mostraram que muitos fungicidas foram eficientes prevenindo a infecção foliar por Alternaria alternata sp. citri. Os fungicidas captan, folpet, maneb, mancozeb, metiram, iprodione e procimidone forneceram excelente proteção. Solel et al. (5) conduziram experimentos por 5 anos com tangelos Minneola e constataram que iprodione foi efetivo reduzindo lesões nos frutos quando aplicado 4 ou 5 vezes em intervalos de 2 semanas, também apresentando bons resultados quando alternado com outros fungicidas como metiram, cobre e mancozeb. A alternância com outros produtos veio como uma estratégia de manejo de resistência de iprodione a Alternaria alternata sp. citri que já era conhecida, pois trabalhos que o próprio autor realizou comprovara que o uso de iprodione, durante 3 anos seguidos, tornou o patógeno resistente no $4^{\circ}$ ano e que a severidade de infecção em folhas inoculadas com isolados resistentes selecionados da Flórida e de Israel, não foi afetada pulverizando iprodione a $250 \mathrm{mg} / \mathrm{litro}$, mas foi reduzida a $500 \mathrm{mg} / \mathrm{litro}$ (4).

O cobre vem sendo a base do programa de controle de $A$. alternata f. sp. citri tanto na Flórida como no Brasil, principalmente pelo baixo custo e por apresentar um controle aceitável da doença; mas no Brasil, ainda não há resultados expressivos no controle desta doença, por isso o objetivo deste trabalho foi estabelecer o melhor produto e dose para o controle da doença no campo.

\section{MATERIAL E MÉTODOS}

O experimento de campo foi realizado na Fazenda Triângulo em Pratânia - SP. O delineamento experimental foi de parcelas subdivididas em blocos, com 10 tratamentos principais e 3 doses (sub-parcelas), com 5 repetições. Os tratamentos consistiram: azoxystrobin, pyraclostrobin, trifloxystrobin, trifloxystrobin + tebuconazole ( 2 aplicações) seguido de 3 aplicações de mancozeb, difenoconazole, trifloxystrobin + propiconazole, iprodione , trifloxystrobin + propineb ( 2 aplicações) seguido de 3 aplicações de oxicloreto de cobre , oxicloreto de cobre + óleo e testemunha (água). As dosagens foram: pulverização de metade da dose recomendada pelo fabricante; pulverização de dose recomendada pelo fabricante; pulverização de uma dose e meia. Foram feitas 5 aplicações, sendo a primeira aplicação no início da brotação, visando reduzir a produção de inóculo sobre a folhagem velha e impedir a infecção de brotações novas, e as aplicações subseqüentes foram em intervalos de 15 dias.

Foram feitas três avaliações de incidência de mancha marrom em frutos [aos 142 dias após a aplicação (DAA), 173 DAA e 203 DAA], onde foi selecionada, ao acaso, uma penca de frutos por planta e determinados o número total de frutos da penca e o número de frutos com lesões.

A colheita foi realizada nos dias 24 e 25 de agosto de 2005 e a produção foi obtida em $\mathrm{Kg}$ por planta, através de pesagem em balança de até $300 \mathrm{Kg}$.

A análise estatística foi realizada pelo programa Estat, comparando-se as médias através do teste de Tukey. Para a análise os dados foram transformados em $\sqrt{\mathrm{x}+0,5}$.

O custo dos ingredientes ativos utilizados no ensaio de campo foi obtido através de cálculo baseando-se na dose utilizada em 1000 litros de calda, onde se obteve o custo por hectare referente a cada dose utilizada, custo em dólares de uma aplicação do tratamento por hectare, de cinco aplicações do tratamento por hectare e do custo das cinco aplicações em caixas por hectare. No momento da análise o valor do dólar estava US\$2,30 e o custo da caixa de murcote era de $\mathrm{R} \$ 8,00$ (Tabela 5).

\section{RESULTADOS E DISCUSSÃO}

A elevada incidência de mancha marrom de alternaria pode ser observada na testemunha nas duas primeiras avaliações, onde $100 \%$ dos frutos apresentavam lesões do patógeno (Tabela 1). Na primeira e na última avaliação todos os tratamentos com fungicidas diferiram estatisticamente da testemunha, demonstrando a necessidade de controle. Na segunda avaliação também não ocorreu diferença estatística entre os tratamentos com fungicidas, porém os tratamentos 1, 2, 3 e 7 foram estatisticamente superiores à testemunha. Este fato pode estar correlacionado às condições climáticas (Figura 1) que favoreceram uma maior pressão de mancha

Tabela 1. Incidência (número de frutos com lesões) de mancha marrom de alternaria em frutos de murcote submetidos à diferentes tratamentos com fungicidas. Pratânia/SP, 2005.

\begin{tabular}{|c|c|c|c|c|c|c|}
\hline \multirow{3}{*}{$\frac{\text { Tratamentos }}{\text { azoxystrobin }}$} & \multicolumn{6}{|c|}{ Avaliações $^{1}$} \\
\hline & \multicolumn{2}{|c|}{$142 \mathrm{DAA}^{2}$} & \multicolumn{2}{|c|}{173 DAA } & \multicolumn{2}{|c|}{203 DAA } \\
\hline & 8,9 & $\mathrm{~d}$ & 42,7 & $\mathrm{~b}$ & 0,0 & $\mathrm{~b}$ \\
\hline pyraclostrobin & 18,0 & bcd & 40,0 & $\mathrm{~b}$ & 10,0 & $\mathrm{~b}$ \\
\hline trifloxystrobin & 11,1 & $\mathrm{~cd}$ & 43,3 & $\mathrm{~b}$ & 6,7 & $\mathrm{~b}$ \\
\hline trifloxys. + tebuconaz.mancozeb & 16,7 & bcd & 51,1 & $a b$ & 0,0 & $\mathrm{~b}$ \\
\hline difenoconazole & 39,6 & bcd & 74,4 & $a b$ & 0,0 & $\mathrm{~b}$ \\
\hline trifloxys.+ propiconazole & 52,8 & $\mathrm{~b}$ & 73,3 & $a b$ & 0,0 & $\mathrm{~b}$ \\
\hline iprodione & 28,9 & bcd & 42,2 & $\mathrm{~b}$ & 1,7 & $\mathrm{~b}$ \\
\hline trifloxys + propineb + cobre & 38,9 & bcd & 75,5 & $\mathrm{ab}$ & 13,3 & $\mathrm{~b}$ \\
\hline cobre + óleo & 49,9 & $\mathrm{bc}$ & 80,0 & $\mathrm{ab}$ & 6,7 & $\mathrm{~b}$ \\
\hline testemunha & 100,0 & $\mathrm{a}$ & 100,0 & $\mathrm{a}$ & 35,5 & $\underline{\mathrm{a}}$ \\
\hline$\overline{\text { C.V. }(\%)^{3}}$ & 84,76 & & 64,36 & & 46,91 & \\
\hline
\end{tabular}

1. Médias seguidas da mesma letra na coluna, não diferem entre si pelo teste de Tukey ao nível de 5\% de probabilidade. Dados originais. Para análise os dados foram transformados em arc sen " $\mathrm{x}+0,5$

2. DAA - dias após a primeira aplicação.

3. Coeficiente de variação em porcentagem. 


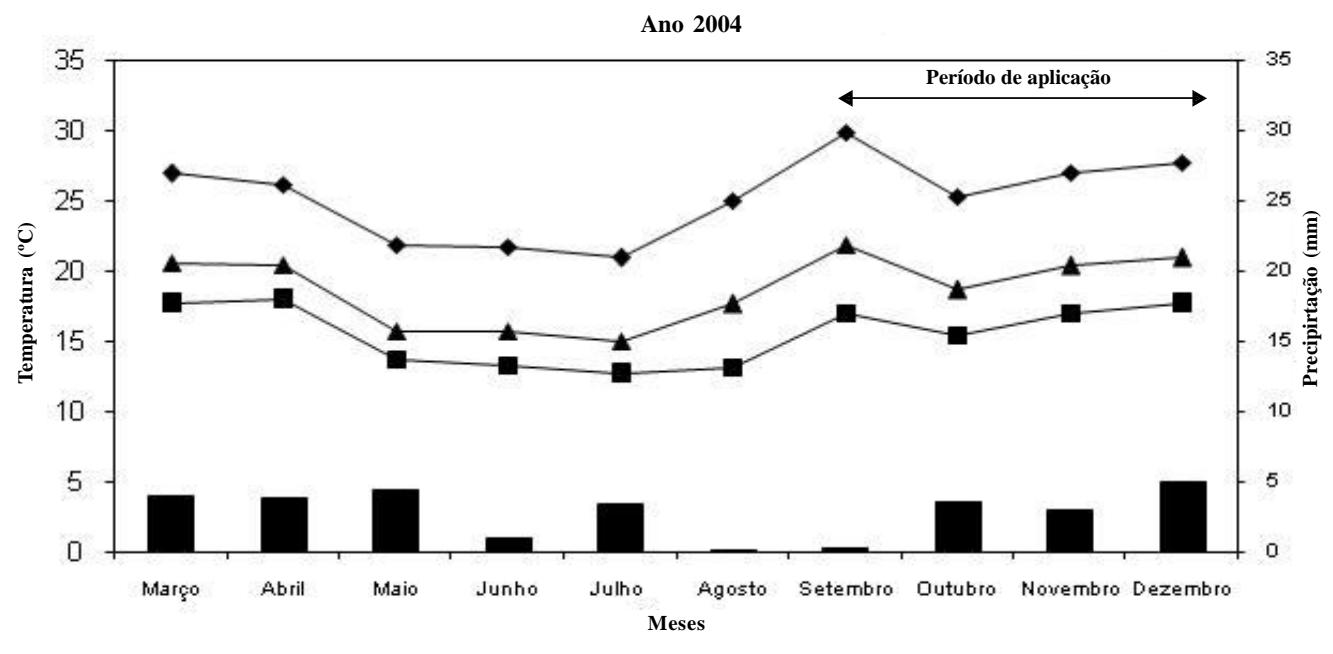

DrecPluvial $\rightarrow$-TempMin $\rightarrow$ Tempmax $\rightarrow$ Templued

Figura 1. Dados climáticos referentes ao ano de 2004, no período em que ocorreu a pesquisa. Fonte: Departamento de Ciências Naturais, FCA/Unesp.

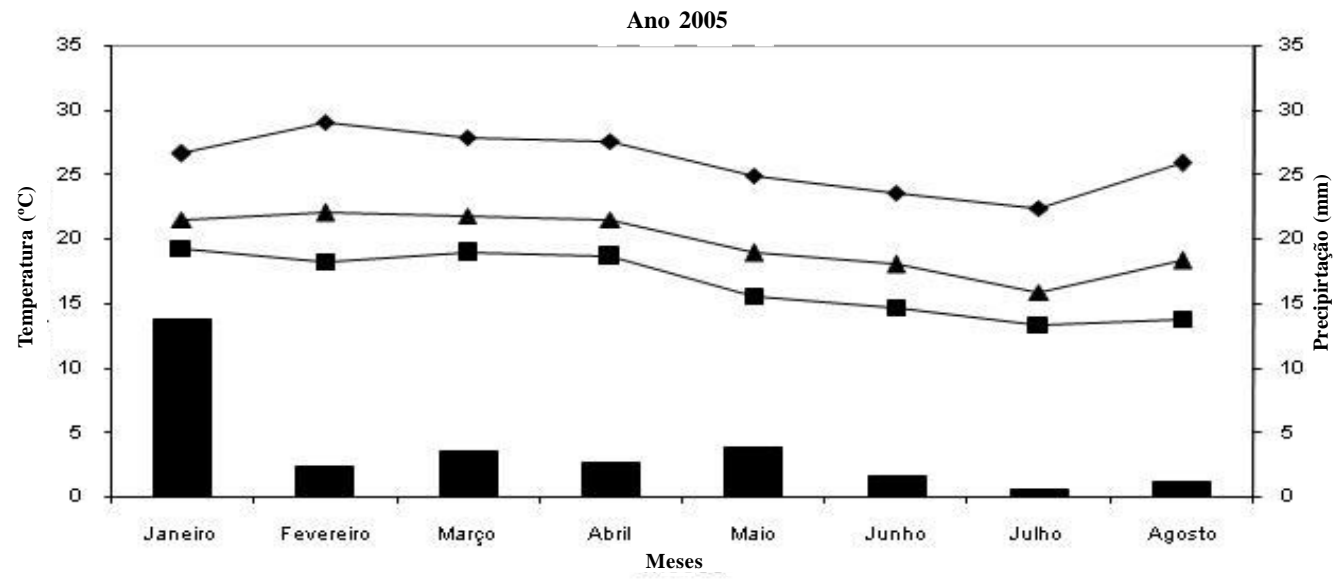

$\longrightarrow$ PrecPluvial $\rightarrow$ TempMin $\rightarrow$ TempMax

Figura 2. Dados climáticos referentes ao ano de 2005, no período em que ocorreu a pesquisa. Fonte: Departamento de Ciências Naturais, FCA/Unesp.

Tabela 2. Efeito de diferentes doses de fungicidas utilizados na incidência (número de frutos com lesões) de Alternaria alternata f. sp. citri em frutos de tangor murcote. Pratânia/SP, 2005.

\begin{tabular}{|c|c|c|c|c|c|c|}
\hline \multirow{3}{*}{$\frac{\text { Doses }}{1}$} & \multicolumn{6}{|c|}{ Avaliações $^{1}$} \\
\hline & \multicolumn{2}{|c|}{142 DAA } & \multicolumn{2}{|c|}{173 DAA } & \multicolumn{2}{|c|}{203 DAA } \\
\hline & 50,1 & $\mathrm{a}$ & 77,6 & $\mathrm{a}$ & 9,7 & $\mathrm{a}$ \\
\hline 2 & 36,0 & b & 63,3 & $a b$ & 6,0 & $\mathrm{~b}$ \\
\hline 3 & 23,3 & $\mathrm{~b}$ & 45,8 & $\mathrm{~b}$ & 6,5 & $\mathrm{ab}$ \\
\hline$\overline{\text { C.V. }(\%)^{3}}$ & \multicolumn{2}{|l|}{76,58} & \multicolumn{2}{|l|}{62,78} & \multicolumn{2}{|c|}{78,36} \\
\hline
\end{tabular}

1. Médias seguidas da mesma letra na coluna, não diferem entre si pelo teste de Tukey ao nível de $5 \%$ de probabilidade. Dados originais. Para análise os dados foram transformados em "x+ 0,5

2. DAA - dias após a primeira aplicação.

3. Coeficiente de variação em porcentagem.

marrom de alternaria no período. Não houve interação entre produtos e doses.

Na Tabela 2 observa-se o efeito de diferentes doses sobre a incidência em frutos de murcote, ficando evidente que a dose recomendada pelo fabricante (dose 2) foi a que melhor resultado apresentou, não diferindo da maior dose em nenhuma das avaliações e sendo superior à menor dose na primeira e terceira avaliações.

Os dados de produção demonstram que todos os tratamentos diferiram da testemunha (Tabela 3), mostrando que, independente do produto utilizado, obteve-se uma produção superior, chegando até ao dobro do número de caixas/ha. Numericamente pode-se notar que o tratamento 8 foi o que apresentou o maior número de caixas/ 
Tabela 3. Avaliação de produtividade de tangor murcote, submetida a diferentes tratamentos com fungicidas visando o controle de mancha marrom de alternaria. Pratânia/SP, 2005.

\begin{tabular}{|c|c|c|c|c|c|c|}
\hline \multirow[b]{3}{*}{ azoxystrobin } & \multicolumn{6}{|c|}{ Produtividade } \\
\hline & \multicolumn{2}{|c|}{$\mathrm{Kg} /$ planta $^{1}$} & \multicolumn{2}{|l|}{$\mathrm{Kg} / \mathrm{ha}$} & \multicolumn{2}{|c|}{ caixa/ha } \\
\hline & 41,89 & $\mathrm{a}$ & 11980,54 & $\mathrm{a}$ & 293,60 & $\mathrm{a}$ \\
\hline pyraclostrobin & 39,78 & a & 11377,08 & $\mathrm{a}$ & 278,85 & $\mathrm{a}$ \\
\hline trifloxystrobin & 37,44 & $\mathrm{a}$ & 10707,84 & $\mathrm{a}$ & 262,45 & a \\
\hline trifloxys. + tebuconaz.mancozeb & 42,36 & a & 12114,96 & $\mathrm{a}$ & 296,90 & $\mathrm{a}$ \\
\hline difenoconazole & 39,68 & a & 11348,48 & a & 278,15 & $\mathrm{a}$ \\
\hline trifloxys.+ propiconazole & 44,05 & a & 12598,30 & a & 308,78 & $\mathrm{a}$ \\
\hline iprodione & 40,18 & $\mathrm{a}$ & 11491,48 & a & 281,65 & $\mathrm{a}$ \\
\hline trifloxys + propineb + cobre & 48,75 & $\mathrm{a}$ & 13942,50 & $\mathrm{a}$ & 341,73 & $\mathrm{a}$ \\
\hline cobre + óleo & 40,73 & $\mathrm{a}$ & 11648,78 & $\mathrm{a}$ & 285,51 & $\mathrm{a}$ \\
\hline testemunha & 21,80 & $\mathrm{~b}$ & 6234,80 & $\mathrm{~b}$ & 152,81 & $\mathrm{~b}$ \\
\hline C.V. $(\%)^{2}$ & 15,13 & & & & & \\
\hline
\end{tabular}

1. Médias seguidas da mesma letra na coluna, não diferem entre si pelo teste de Tukey ao nível de $5 \%$ de probabilidade. Dados originais. Para análise os dados foram transformados em " $x+0,5$ 2. Coeficiente de variação em porcentagem.

Tabela 4. Produtividade de tangor murcote, submetida a diferentes tratamentos com fungicidas e diferentes doses visando o controle de mancha marrom de alternaria. Pratânia/SP, 2005.

\begin{tabular}{|c|c|c|c|}
\hline \multirow[b]{2}{*}{ Doses } & \multicolumn{3}{|c|}{ Produtividade } \\
\hline & $\mathrm{Kg} /$ planta $^{1}$ & $\mathrm{Kg} / \mathrm{ha}$ & caixa/ha \\
\hline 1 & 44,59 a & 12752,74 & 312,57 \\
\hline 2 & $37,31 \quad b$ & 10670,66 & 261,53 \\
\hline 3 & $43,06 \mathrm{ab}$ & 12315,16 & 301,84 \\
\hline C.V. $(\%)^{2}$ & 19,51 & & \\
\hline
\end{tabular}

1. Médias seguidas da mesma letra na coluna, não diferem entre si pelo teste de Tukey ao nível de $5 \%$ de probabilidade. Dados originais. Para análise os dados foram transformados em " $\mathrm{x}+0,5$

2. Coeficiente de variação em porcentagem.

ha, seguido do tratamento 6 com 308,78 caixas/ha. Porém, ao verificar o custo de aplicação em caixas por tratamento (Tabela 5), nota-se que o tratamento 6 seria o mais rentável, pois descontando o custo da aplicação sua produtividade em caixas/ha foi maior.

$\mathrm{Na}$ Tabela 4, onde verifica-se a diferença entre doses dos produtos utilizados, a dose recomendada pelo fabricante mostrouse estatisticamente superior à menor dose e não apresentou diferença estatística para a maior dose.

As estrubilurinas pyraclostrobin, azoxystrobin e o oxicloreto de cobre utilizados tanto em mistura com óleo vegetal como após duas aplicações da mistura trifloxystrobin + propinebe, promoveram um bom controle quando comparado com a testemunha, confirmando os resultados obtidos por Alka Bathia \& Timmer (1). Na Flórida as estrobilurinas são altamente efetivas e recomendadas para o controle da mancha de alternaria (10).

Tebuconazole e outros fungicidas triazóis não foram efetivos em estudos na Flórida e em Israel (7). Mancozeb demonstrou bom controle na África do Sul (3).

Experimentos de campo conduzidos por vários anos por Solel et al. (5) demonstraram a eficácia de iprodione no controle da mancha de alternaria em frutos, aumentando a porcentagem de frutos de primeira classe de qualidade; neste experimento iprodione demonstrou controle satisfatório diferindo da testemunha, porém não diferiu estatisticamente dos outros produtos avaliados, mas o desenvolvimento da resistência de iprodione a mancha de alternaria em Israel limitou o seu uso para 1 ou 2 aplicações por ano (4).

$\mathrm{O}$ custo dos ingredientes ativos utilizados no manejo da mancha marrom de alternaria é um fator importante na tomada de decisão, uma vez vencido os demais parâmetros técnicos que norteiam esta escolha. Dos produtos utilizados neste experimento verifica-se que o tratamento 6 (trifloxystrobin + propiconazole), efetuado em 5 aplicações, mostrou-se o mais adequado para a relação custo/ benefício, pois mostrou-se tecnicamente igual ao tratamento 8 (trifloxystrobin + propineb, em duas aplicações e oxicloreto de cobre em 3 aplicações) (Tabela 6). O que se observou neste experimento é que, independente do ingrediente ativo utilizado, todos os tratamentos avaliados mostraram-se economicamente viáveis, pois as perdas provocadas pela doença foram superiores a $50 \%$ se comparadas com os tratamentos fungicidas.

Os produtos iprodione, trifloxystrobin, azoxystrobin, pyraclostrobin e cobre (10), utilizados como padrão na Flórida, também apresentaram resultados satisfatórios em nossa região, sendo, então, o fator preço que irá condicionar a escolha do produto.

A mistura trifloxystrobin com propiconazole é rentável na comparação produtividade/custo, sendo recomendável para o controle de mancha marrom de alternaria. A dose recomendada pelo fabricante apresentou os melhores resultados. 
Tabela 5. Custo dos fungicidas utilizados no controle de mancha marrom de alternaria em tangor murcote.

\begin{tabular}{|c|c|c|c|}
\hline Ingrediente ativo & Dose/1000L & Custo em $\mathbf{R} \$ /$ ha & $\mathbf{R} \$ /$ litro/Kg \\
\hline \multirow[t]{3}{*}{ trifloxystrobin } & $37,5 \mathrm{~g}$ & 64,35 & $\mathrm{R} \$ 750,00$ \\
\hline & $75 \mathrm{~g}$ & 128,7 & \\
\hline & $112,5 \mathrm{~g}$ & 193,05 & \\
\hline \multirow[t]{3}{*}{ azoxystrobin } & $50 \mathrm{~g}$ & 51,48 & $\mathrm{R} \$ 450,00$ \\
\hline & $100 \mathrm{~g}$ & 102,96 & \\
\hline & $150 \mathrm{~g}$ & 154,44 & \\
\hline \multirow[t]{3}{*}{ pyraclostrobin } & $100 \mathrm{~mL}$ & 34,32 & $\mathrm{R} \$ 150,00$ \\
\hline & $200 \mathrm{~mL}$ & 68,64 & \\
\hline & $300 \mathrm{~mL}$ & 102,96 & \\
\hline \multirow[t]{3}{*}{ difenoconazole } & $175 \mathrm{~mL}$ & 72,07 & $\mathrm{R} \$ 180,00$ \\
\hline & $350 \mathrm{~mL}$ & 144,14 & \\
\hline & $525 \mathrm{~mL}$ & 216,21 & \\
\hline \multirow[t]{3}{*}{ trifloxystrobin + tebuconazole } & $250 \mathrm{~mL}$ & 68,64 & $\mathrm{R} \$ 120,00$ \\
\hline & $500 \mathrm{~mL}$ & 137,28 & \\
\hline & $750 \mathrm{~mL}$ & 205,92 & \\
\hline \multirow[t]{3}{*}{ trifloxystrobin + propiconazole } & $37,5 \mathrm{~mL}$ & 9,43 & $\mathrm{R} \$ 110,00$ \\
\hline & $75 \mathrm{~mL}$ & 18,87 & \\
\hline & $112,5 \mathrm{~mL}$ & 28,29 & \\
\hline \multirow[t]{3}{*}{ trifloxystrobin + propinebe } & $500 \mathrm{~g}$ & 22,88 & $\mathrm{R} \$ 20,00$ \\
\hline & $1000 \mathrm{~g}$ & 45,76 & \\
\hline & $1500 \mathrm{~g}$ & 68,64 & \\
\hline \multirow[t]{3}{*}{ oxicloreto de cobre + óleo } & $1000 \mathrm{~mL}+500 \mathrm{~mL}$ & 17,16 & $R \$ 10,00+R \$ 5,00$ \\
\hline & $2000 \mathrm{~mL}+1000 \mathrm{~mL}$ & 34,32 & \\
\hline & $3000 \mathrm{~mL}+1500 \mathrm{~mL}$ & 51,48 & \\
\hline \multirow[t]{3}{*}{ oxicloreto de cobre } & $1000 \mathrm{~mL}$ & 11,44 & $\mathrm{R} \$ 10,00$ \\
\hline & $2000 \mathrm{~mL}$ & 22,88 & \\
\hline & $3000 \mathrm{~mL}$ & 34,32 & \\
\hline \multirow[t]{3}{*}{ mancozeb } & $1000 \mathrm{~g}$ & 12,58 & $\mathrm{R} \$ 11,00$ \\
\hline & $2000 \mathrm{~g}$ & 25,16 & \\
\hline & $3000 \mathrm{~g}$ & 37,75 & \\
\hline \multirow[t]{3}{*}{ iprodione } & $75 \mathrm{~mL}$ & 54,34 & $\mathrm{R} \$ 95,00$ \\
\hline & $150 \mathrm{~mL}$ & 108,68 & \\
\hline & $225 \mathrm{~mL}$ & 163,02 & \\
\hline
\end{tabular}


Tabela 6. Custo das aplicações utilizadas no controle de mancha marrom de alternaria, preço de uma aplicação por hectare, preço das cinco aplicações efetuadas e custo transformado em caixas de tangor murcote por hectare.

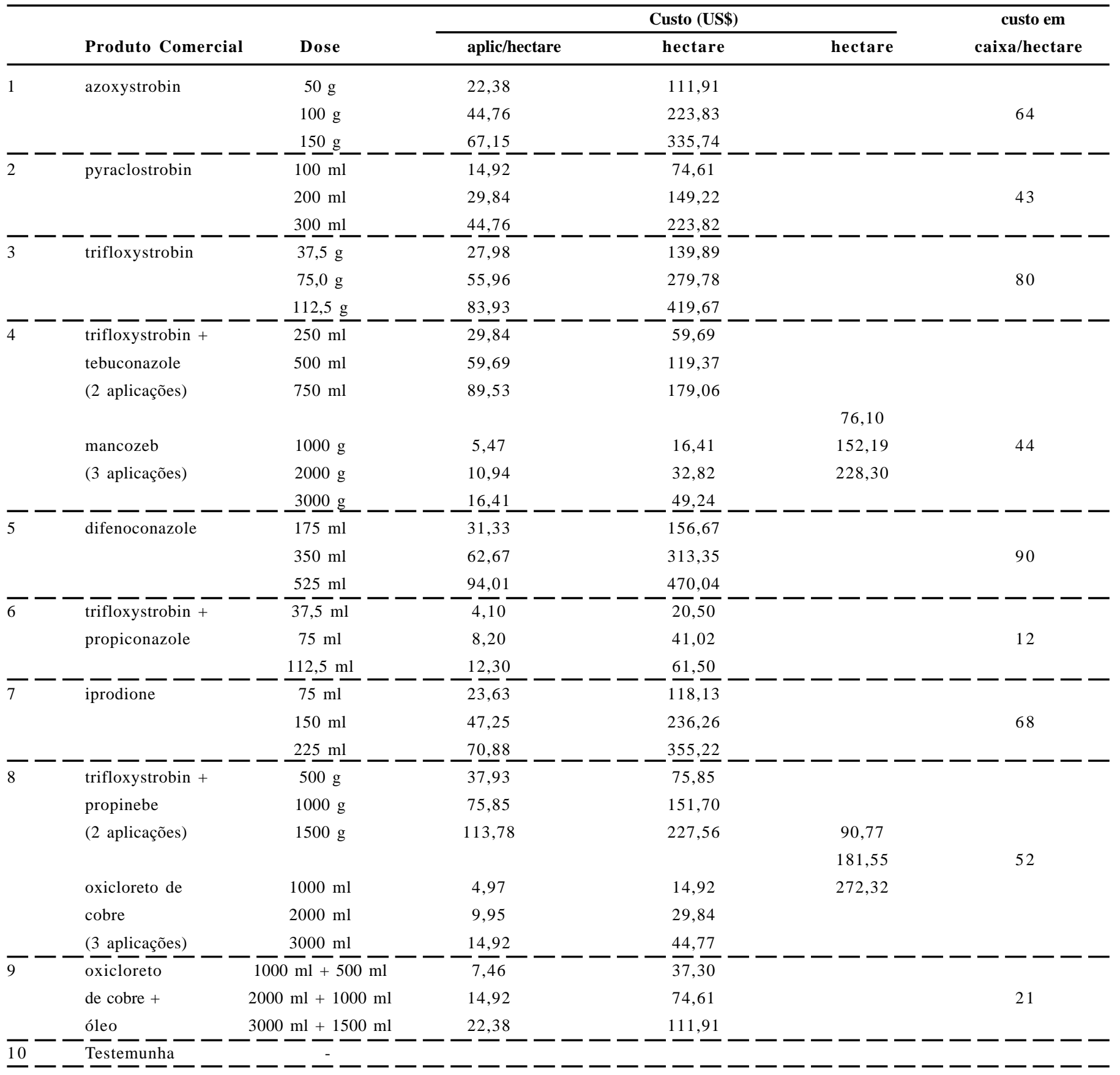

\section{REFERÊNCIAS BIBLIOGRÁFICAS}

1. Alka Bhatia; Timmer, L.W. Evaluation of fungicides for control of Alternaria brown spot on Murcotts, 2001. Disponível em <http:// www.crec.ifas.ufl.edu/timmer/alternariafungicidereports.htm>. Acesso em: 28 jan. 2006.

2. Prates, H.S. Mancha de Alternaria nas tangerinas. Revista Coopercitrus, Campinas, n.205, p. 12-14, 2004.

3. Schutte, G.C.; Lesar, K.H.; Pelser, P.duT., Swart, S.H. The use of tebuconazole for control of Alternaria alternate on 'Minneola' tangelos and its potential to control post-harvest decay when applied as a pre-harvest spray. Proceeding of the Internacional Society of Citriculture, Orlando, n.7, p. 1070-1074, 1992.

4. Solel, Z.; Timmer, L.W.; Kimchi, M. Iprodione resistence of Alternaria alternate pv. Citri from Minneola Tangelo in Israel and Florida. Plant Disease, St. Paul, v. 80, n. 3, p. 291-293, 1996
5. Solel, Z.; Oren, Y. Kimchi, M.Control of Alternaria brown spot of Minneola tangelo with fungicides. Crop Protection, Oxford, v. 16, n. 7, p. 659-664, 1997.

6. Swart, S.H.; Wingfield, J.; Swart, W.J.; Schutte, G.C. Chemical control of Alternaria brown spot on Minneola tangelo in South Africa. Annals of Applied Biology, Bognor Regis, v. 133, n.1, p. 17-30, 1998.

7. Timmer, L.W.; Zitko, S.E. Evaluation of fungicides for control of Alternaria brown spot and citrus scab. Proceedings of the Florida State Horticultural Society, St. Paul, n.110, p. 71-76, 1997.

8. Timmer, L. W.; Garnsey, S.M.; Graham, J.H. Compendium of citrus diseases. 2.ed. Minnesota: APS, 2000. 128p.

9. Timmer, L.W. Alternaria Brown Spot. Disponível em:<http:// www.crec.ifas.ufl.edu/timmer>. Acesso em: 24 set. 2004.

10. Timmer, L.W.; Roberts, M.J.; Chung, K.-R. Alternaria brown spot. In: Timmer, L.W., Rogers, M.E. Buker, R.S. Florida citrus pest management guide. Gainesville: FL Publication, 2005. p. 43-43. 